\title{
Analyse quantitative des composés phénoliques d'une endémique algérienne Scabiosa Atropurpurea sub. Maritima L.
}

Manuscrit reçu le 21 juillet 2017 et accepté le 21 février 2018

Fahima Ali-RACHEdI $^{*}{ }^{(1.2)}$, Souad MERAGHNI ${ }^{(3)}$, Nourhène TouAIBIA ${ }^{(1)}$ et Sabrina $^{(1)}$

${ }^{(1)}$ Univ Souk Ahras, Faculté des Science et la Technologie, PB 1553, 41000 Souk Ahras, Algérie

${ }^{(2)}$ Laboratoire de chimie Organique Appliquée, Université de Annaba, 23000 Annaba, Algérie

${ }^{(3)}$ Laboratoire de biologie végétale et environnement, université de Annaba, B.P 12 Annaba, Algérie

\section{Résumé:}

Scabiosa Atropurpurea sub. Maritima L. est une plante de la famille des Dipsacacée utilisée dans la médecine traditionnelle pour le traitement de certaines maladies de peau, en particulier la gale. Notre étude porte sur l'évaluation par dosage spectrophotométrique des polyphénols et des flavonoïdes. Le dosage des phénols totaux montre que la teneur la plus élevée des phénols a été mesurée dans l'extrait méthanolique, avec une valeur égale à 1,303 mg EAG/g ES, suivi par les extraits hexanique et chloroformique respectivement. Le dosage des flavonoïdes a révélé que l'extrait méthanolique renferme un maximum de flavonoïdes, avec un taux de 0,613 mg EQ/g ES. Nous avons également effectué les dosages des tanins et des anthocyanes.

Mots clés: Scabiosa Atropurpurea L, screening phytochimique, phénols totaux- flavonoïdes.

\section{Abstract:}

Scabiosa Atropurpurea sub. Maritima L. is a plant of the family of Dipsacaceae used in traditional medicine for the treatment of certain skin diseases, especially scabies. Our study focuses on the spectrophotometric assay of polyphenols and flavonoids. The determination of the total phenols shows that the highest content of the polyphenols was measured in the methanol extract with a value equal to $1,303 \mathrm{mg}$ EAG / g ES, followed by hexane and chloroform extracts, respectively. The flavonoid dosage revealed that the methanolic extract contained a maximum of flavonoids with a level of 0,613 $\mathrm{mg}$ EQ / $\mathrm{g}$ ES. We also performed the assays of tannins and anthocyanins.

\footnotetext{
*Auteur correspondant : fahima_alirachedi@yahoo.fr
} 
Keywords: Scabiosa Atropurpurea L., phytochemical screening - total phenols - flavonoids.

\section{Introduction}

L'espèce que nous avons choisie est : Scabiosa Atropurpurea sub.maritima L., c'est une plante médicinale de la famille des Dipsacaceae, reconnue pour son utilisation dans le traitement de certaines maladies de peau. Généralement, la scabieuse est employée pour ses vertus stomachiques, expectorantes, toniques, vulnéraires, astringentes, dépuratives et digestives. Elle permet de lutter contre les diverses affections de la peau comme, la gale, les eczémas, les dartres et les prurits [1]. Cette plante est également efficiente pour traiter les problèmes d'aphtes. D'autre part, la scabieuse a démontré une réelle efficacité dans le traitement de certaines maladies des voies respiratoires, notamment la grippe, la bronchite et l'asthme [1].

La famille des Dipsacacées est constituée de plantes dicotylédones, elle comprend 150 espèces réparties en 6 à 14 genres. Ce sont des plantes herbacées annuelles, bisannuelles ou pérennes originaires des régions tempérées de l'hémisphère nord [2]. Ce sont des plantes herbacées, parfois arbustives, souvent de type xérophyte, réparties en Afrique et en Eurasie. Les feuilles sont simples, parfois très découpées, le plus souvent opposées. Les fleurs ont un calice peu développé muni d'un épicalice appelé involucelle. Le fruit est un akène, généralement surmonté d'un épicalice accrescent [2].

À ce jour, aucune présence de substances toxiques nocives à l'organisme n'a été décelée dans la scabieuse. Le présent travail, a pour but d'étudier la teneur en composés phénoliques par dosage spectrophotométrique des extraits méthanolique, chloroformique et hexanique d'une endémique algérienne Scabiosa Atropurpurea sub. maritima L.

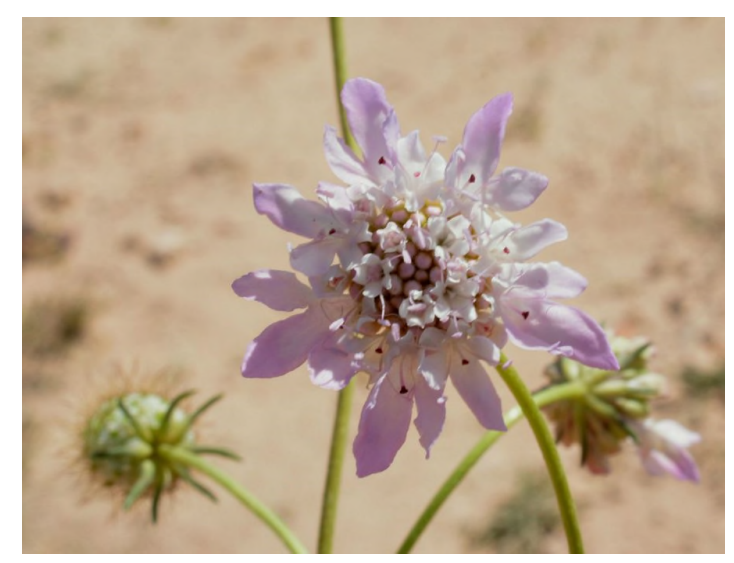

Fig. 1 : Scabiosa atropurpurea subsp. maritima (L.) Greuter \& Burdet (photo: Chatelain) 


\section{Matériel et méthodes}

\subsection{Matériel végétal}

L'espèce que nous avons étudiée est : Scabiosa Atropurpurea sub Maritima L., c'est une plante médicinale de la famille des Dipsacaceae, reconnue pour son utilisation dans le traitement de certaines maladies de peau. Cette espèce a été récolté au mois de juillet dans la région de cap de garde à Annaba et identifiée par le Dr Gérard DE BELAIR; un herbier français demeurant à Annaba.

Les feuilles de cette plante ont été découpées, séchées à température ambiante à l'abri de la lumière, ensuite réduite en poudre. La poudre a été macérée dans trois solvants de polarité croissante, hexane chloroforme et méthanol, pendant $48 \mathrm{~h}$, filtrée et concentrée par évaporation rotative. Les extraits bruts obtenus ont été conservés à $-4^{\circ} \mathrm{C}$.

\subsection{Dosage des polyphénols totaux}

Le dosage des polyphénols totaux a été déterminé par spectrophotométrie, selon la méthode colorimétrique utilisant le réactif de Folin-Ciocalteu [3]. Ce dosage est basé sur la quantification de la concentration totale de groupements hydroxyles présents dans l'extrait.

Le protocole utilisé est basé sur celui décrit par (Singleton et Ross, 1965) [4] en y apportant quelques modifications. Brièvement, dans des tubes à hémolyse en verre, un volume de $200 \mu \mathrm{l}$ de chaque extrait a été ajouté, avec un mélange de $1 \mathrm{ml}$ de réactif Folin-Ciocalteu dilué 10 fois, et $800 \mu \mathrm{l}$ d'une solution de carbonate de sodium à 7,5\%. Les tubes sont agités et conservés pendant $30 \mathrm{~min}$. L'absorbance est lue à $765 \mathbf{~ n m}$.

Une courbe d'étalonnage a été réalisée en parallèle dans les mêmes conditions opératoires en utilisant l'acide gallique à différentes concentrations (0 à $1000 \mu \mathrm{g} / \mathrm{ml})$.

\subsection{Dosage des flavonoïdes totaux}

La quantification des flavonoïdes a été effectuée par une méthode basée sur la formation d'un complexe très stable, entre le chlorure d'aluminium et les atomes d'oxygène présent sur les carbones 4 et 5 des flavonoïdes [5].

Le protocole utilisé est basé sur celui décrit par (Zhishen et al., 1999) [6] et (Kim et al., 2003)

[7], avec quelques modifications.

Dans un tube à hémolyse en verre, $400 \mu \mathrm{l}$ d'extrait, ou d'étalon, ou de l'eau distillée pour le témoin, ont été ajoutés à $120 \mu \mathrm{l}$ de $\mathrm{NaNO}_{2}$ à $5 \%$. Après 5 minutes, $120 \mu \mathrm{l} \mathrm{d}^{\prime} \mathrm{AlCl}_{3}$ à $10 \%$ on été additionés, et le milieu est mélangé vigoureusement. Après 6 minutes, un volume de 800 $\mu \mathrm{l}$ de $\mathrm{NaOH}$ à $1 \mathrm{M}$ a été ajouté au milieu. L'absorbance est lue immédiatement à $\mathbf{5 1 0} \mathbf{~ n m}$ contre le témoin. Une solution méthanolique de quercétine a été préparée. Des solutions filles 
préparées à partir de la solution mère à différentes concentrations comprises entre 0 et 1000 $\mu \mathrm{g} / \mathrm{ml}$, permettront de tracer la courbe d'étalonnage.

\subsection{Dosage des tanins condensés}

Nous avons adopté la méthode à la vanilline avec l'HCl. Cette méthode dépend de la réaction de la vanilline avec le groupement flavonoïde terminal des TCs et la formation de complexes rouges $[8,9]$, cela s'explique par la propriété des tanins à se transformer en anthocyanidols de couleur rouge par réaction avec la vanilline [10]. La teneur en tanins condensés a été déterminée par la méthode de vanilline décrite par (Julkunen-Titto, 1985) [11].

Un volume de $50 \mu \mathrm{l}$ de chaque extrait a été ajouté à $1500 \mu \mathrm{l}$ de la solution vanilline/méthanol à $4 \%$, puis mélangé vigoureusement. Ensuite, un volume de $750 \mu \mathrm{l}$ de l'acide chlorhydrique concentré $(\mathrm{HCl})$ a été additionné. Le mélange obtenu est laissé réagir à température ambiante pendant 20 min. L'absorbance est mesurée à $\mathbf{5 5 0} \mathbf{~ n m}$ contre un blanc. Différentes concentrations comprises entre 0 et $1000 \mu \mathrm{g} / \mathrm{ml}$ préparées à partir d'une solution mère de la catéchine, permettront de tracer la courbe d'étalonnage.

\subsection{Dosage des anthocyanes}

La méthode spectrophotométrique des $\mathrm{pH}$ différentiels permet une mesure rapide et précise des anthocyanes totaux même en présence de pigments polymérisés dégradés et d'autres composés interférents [12]. Elle est basée sur la détermination de l'absorbance des solutions extractives diluées avec des solutions tampon de $\mathrm{pH}=1$ et de $\mathrm{pH}=4,5$.

Les anthocyanes se transforment réversiblement sous l'influence du $\mathrm{pH}$. Le changement structurel associé avec la modification des chromophores détermine la couleur différente des solutions des anthocyanes en fonction du $\mathrm{pH}$. La forme colorée (oxonium) prédomine à $\mathrm{pH} 1$ et la forme incolore (hémiacétal) à pH 4,5.

Le protocole adopté est basé sur celui décrit par (Giusti et al., 2001) [12].

\section{Résultats et discussion}

Les analyses quantitatives des phénols totaux, ont été déterminées à partir de l'équation de la régression linéaire de la courbe d'étalonnage, tracée en utilisant l'acide gallique comme standard (Figure 2). Les valeurs obtenues sont exprimées en mg EAG/g ES et en mg EAG/g MS (Tableau 1). 


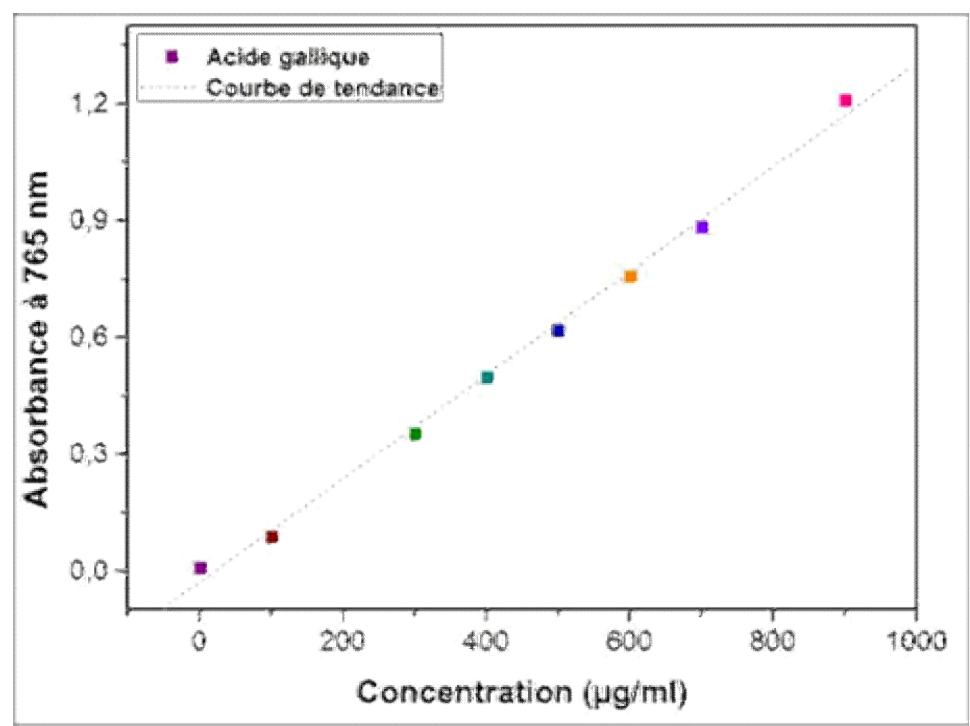

Fig. 2 : Courbe d'étalonnage de l'acide gallique

Tableau 1 : Résultats du dosage des polyphénols

\begin{tabular}{|c|c|c|c|c|}
\hline \multirow{2}{*}{ Extrait } & \multicolumn{2}{|c|}{ Quantité des polyphénols totaux } & \multirow{2}{*}{$\begin{array}{c}\text { Équation de la } \\
\text { courbe }\end{array}$} & \multirow{2}{*}{$\mathbf{R}^{\mathbf{2}}$} \\
\cline { 2 - 3 } & $($ en mg EAG/g ES) & (en mg EAG/g MS $)$ & \multirow{2}{*}{ Abs $=0,001[\mathrm{AG}]-$} & \multirow{2}{*}{0,996} \\
\hline Méthanol & 1,303 & 1,675 & 0,031 & \\
\hline Hexane & 0,924 & 1,164 & 0,764 & \\
\hline Chloroforme & 0,475 & &
\end{tabular}

AG : acide gallique, EAG : équivalent d'acide gallique, $\mathbf{E S}$ : extrait sec, MS : matière sèche

Les teneurs en polyphénols totaux des différentes fractions varient entre 0,475 et $1,303 \mathrm{mg}$ EAG/g ES. La concentration la plus élevée des phénols a été mesurée dans l'extrait méthanolique, avec un taux de 1,303 $\mathrm{mg}$ EAG/g ES, par rapport aux extraits hexanique et chloroformique, où nous enregistrons des teneurs de l'ordre de 0,924 et 0,475 mg EAG/g ES respectivement.

D’après ces résultats, on déduit que le contenu phénolique dans les extraits examinés, dépend de la polarité du solvant utilisé pour l'extraction.

Les taux des flavonoïdes des extraits ont été calculés à partir de la courbe d'étalonnage, tracée en utilisant la quercétine comme standard (Figure 3). Ils sont exprimés en termes de $\mathrm{mg} \mathrm{EQ/g} \mathrm{ES} \mathrm{et} \mathrm{mg} \mathrm{EQ/g} \mathrm{MS} \mathrm{(Tableau} \mathrm{2).}$ 


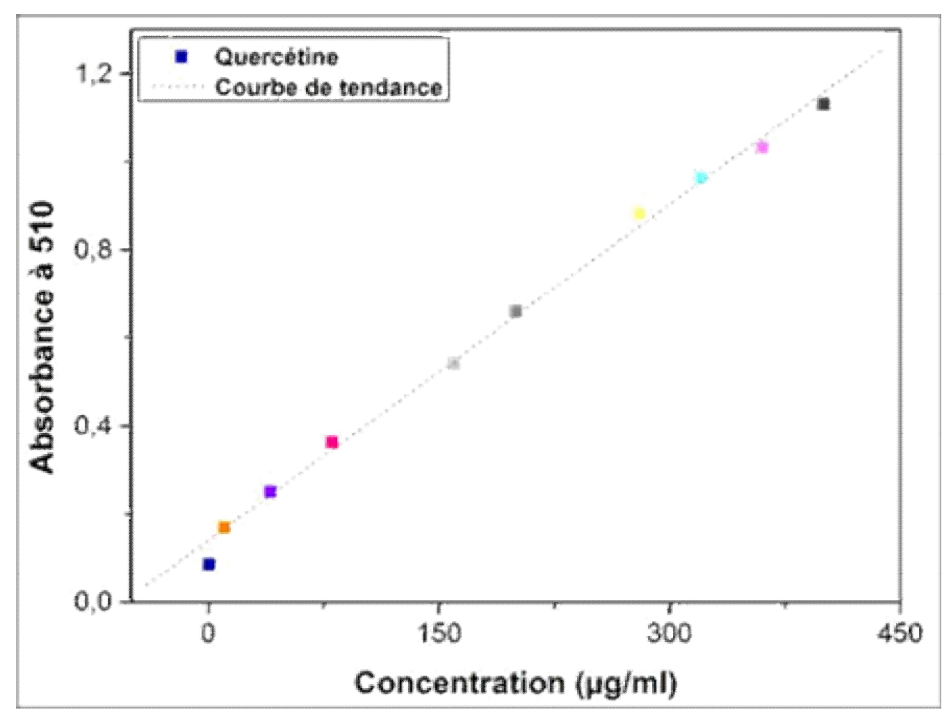

Fig. 3 : Courbe d'étalonnage de la quercétine

Tableau 2 : Résultats du dosage des flavonoïdes

\begin{tabular}{|c|c|c|c|c|}
\hline \multirow{2}{*}{ Extrait } & \multicolumn{2}{|c|}{ Quantité des flavonoïdes totaux } & \multirow{2}{*}{$\begin{array}{l}\text { Équation de la } \\
\text { courbe }\end{array}$} & \multirow{2}{*}{$\mathrm{R}^{2}$} \\
\hline & (en $\mathrm{mg} E Q / g \mathrm{ES})$ & (en $\mathrm{mg} \mathrm{EQ} / \mathrm{g} \mathrm{MS}$ ) & & \\
\hline Méthanol & 0,613 & 7,374 & \multirow{3}{*}{$\begin{array}{c}\mathrm{Abs}=0,002[\mathrm{Que}]+ \\
0,139\end{array}$} & \multirow{3}{*}{0,995} \\
\hline Hexane & 0,148 & 0,186 & & \\
\hline Chloroforme & 0,058 & 0,093 & & \\
\hline
\end{tabular}

Que : quercétine, EQ : équivalent de quercétine, $\mathbf{E S}$ : extrait sec, MS : matière sèche.

Les résultats présentés dans le Tableau 2 montrent que les teneurs en flavonoïdes totaux varient considérablement entre les différents extraits. L'extrait méthanolique enregistre un maximum de flavonoïdes $(0,613 \mathrm{mg} \mathrm{EQ/g} \mathrm{ES})$, suivi par l'extrait hexanique qui renferme des teneurs plus faibles $(0,148 \mathrm{mg} E Q / g$ ES). Tandis que la plus basse concentration des flavonoïdes a été mesurée dans l'extrait chloroformique (0,058 mg EQ/g ES).

La concentration des flavonoïdes dans les extraits de la plante, dépend de la polarité des solvants utilisés dans la préparation des extraits [13].

La teneur en tanins a été à partir de la courbe d'étalonnage, tracée en utilisant la catéchine comme standard. Les résultats ont été déterminés à partir de l'équation de la régression linéaire de la courbe d'étalonnage (Figure 4), et sont exprimés en mg EC/g ES et mg EC/g MS (Tableau 3). 


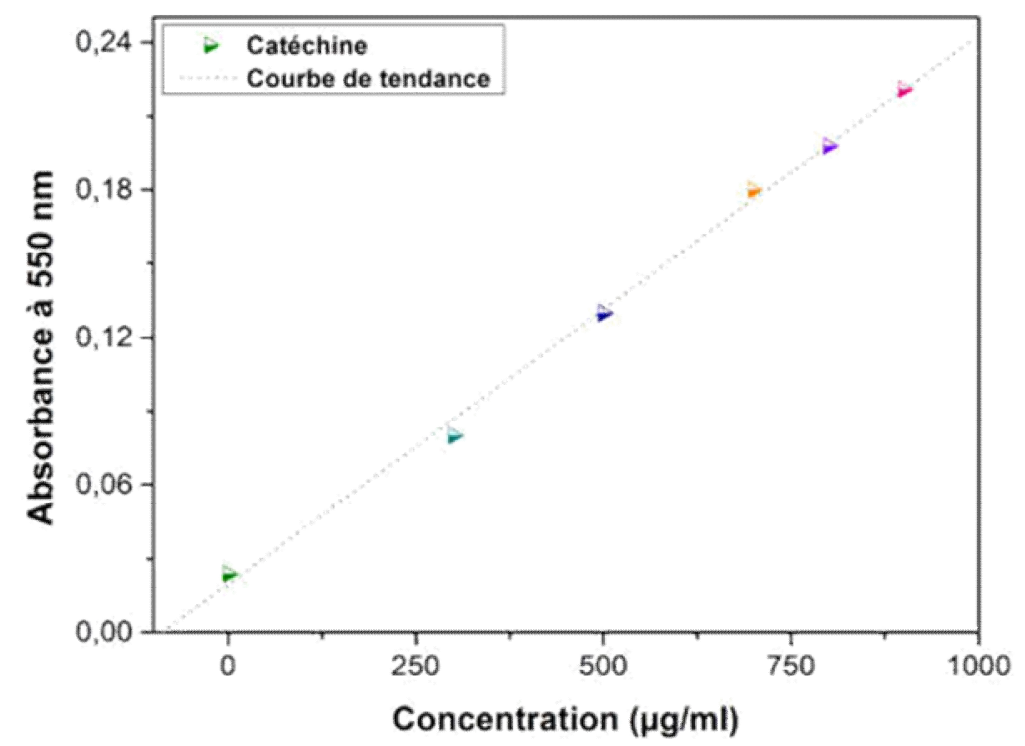

Fig. 4 : Courbe d'étalonnage de la catéchine

Tableau 3 : Résultats du dosage des tanins

\begin{tabular}{|c|c|c|c|c|}
\hline \multirow{2}{*}{ Extrait } & \multicolumn{2}{|c|}{ Quantité des tanins totaux } & \multirow{2}{*}{$\begin{array}{c}\text { Équation de la } \\
\text { courbe }\end{array}$} & \multirow{2}{*}{$\mathbf{R}^{\mathbf{2}}$} \\
\cline { 2 - 3 } & (en mg EC/g ES) & (en mg EC/g MS $)$ & \multirow{2}{*}{$\begin{array}{c}\text { Abs }=0,0002[\mathrm{Cat}]+ \\
0,019\end{array}$} & 0,997 \\
\hline Méthanol & 0,560 & 6,736 & 2,205 & \\
\hline Hexane & 1,750 & 2,117 & \\
\hline Chloroforme & 1,315 & & & \\
\hline
\end{tabular}

Cat : catéchine, EC : équivalent de catéchine, ES : extrait sec, MS : matière sèche.

Ce dosage révèle que la fraction méthanolique renferme les plus importantes teneurs en tanins condensés, avec une valeur de 6,736 mg EC/g MS. En revanche, les fractions hexanique et chloroformique ont enregistré des teneurs plus faibles ; leur concentrations ont atteint 2,205 et 1,315 mg EC/g MS respectivement. Cette variation peut s'expliquer par le fait que l'extraction des tanins condensés, dépend de leur nature chimique, du solvant utilisé et des conditions opératoires [14].

La présence des tanins suggère la capacité de notre plante à joué un rôle majeur en tant qu'agent antimicrobien et antioxydant [15].

La méthode spectrophotométrique de $\mathrm{pH}$ différentiel nous a permis d'aboutir aux quantités d'anthocyanes monomères totaux. Les valeurs mesurées des anthocyanes sont exprimées en $\mathrm{mg} \mathrm{EC}_{3} \mathrm{G} / \mathrm{g}$ ES et $\mathrm{mg} \mathrm{EC}_{3} \mathrm{G} / 100 \mathrm{~g} \mathrm{MS}$, et elles sont regroupées dans le (Tableau 4). 
Tableau 4 : Résultats du dosage des anthocyanes

\begin{tabular}{|c|c|c|c|}
\hline \multirow{2}{*}{ Extrait } & \multicolumn{3}{|c|}{ La concentration en anthocyanes monomères totaux } \\
\cline { 2 - 4 } & $(\mathrm{en} \mathrm{mg} / \mathrm{ml})$ & $\left(\mathrm{en} \mathrm{mg} \mathrm{EC}_{3} \mathrm{G} / \mathrm{g} \mathrm{ES}\right)$ & $\left(\mathrm{en} \mathrm{mg} \mathrm{EC}_{3} \mathrm{G} / 100 \mathrm{~g} \mathrm{MS}\right)$ \\
\hline Méthanol & 3,105 & 1,552 & 1867,056 \\
\hline Hexane & 0,40 & 0,20 & 25,20 \\
\hline Chloroforme & 1,302 & 0,651 & 104,811 \\
\hline
\end{tabular}

$\mathbf{E C}_{\mathbf{3}} \mathbf{G}$ : équivalent de cyanidine-3-glycosylée, $\mathbf{E S}$ : extrait sec, $\mathbf{M S}$ : matière sèche.

D'après les résultats obtenus, il apparaît que l'extrait méthanolique est le plus riche en anthocyanes, avec une teneur de 1867,056 mg EC $3 \mathrm{G} / 100 \mathrm{~g}$ MS. Tandis que les extraits chloroformique et hexanique renferment des teneurs largement inférieurs a celle de l'extrait méthanolique; soit 104,811 et 25,2 $\mathrm{mg} \mathrm{EC}_{3} \mathrm{G} / 100 \mathrm{~g}$ MS respectivement.

La teneur élevée en anthocyanes contenue dans la fraction méthanolique, permet d'expliquer en grande partie sa meilleure capacité antioxydante par rapport aux deux autres fractions.

\section{Conclusion:}

La présente étude s'est proposée de réaliser la quantification de composés polyphénoliques par spectrophotométrie des extraits sec des feuilles d'une plante endémique de la flore algérienne utilisées dans le traitement des maladies de la peau. Les résultats obtenus nous permettent de déduire que l'emploi récurent de cette plante serait le fait de la manifestation leur richesse relative en constituants polyphénoliques.

\section{Références}

[1] Girre, L. Connaître et Reconnaître les Plantes Médicinales Ouest France, 1980, 25(7), 332334.

[2] Al-Qudah, M. A.; Muhaidat, R.; Trawenh, I. N.; Al Jaber, H. I; Abu Zarga, M. H.; Abu Orabi, S. T., Jordan Journal of Chemistry, 2012, 7 (3), 287-295.

[3] V.L.Singleton, R.Orthofer, R.M.Lamuela-Raventos, "Analysis of total phenols and other oxidant substrates and antioxidants by means of Folin-Ciocalteu reagent" Methods Enzymol., 1999, Vol. (299), page: 152.

[4] V.L.Singleton, J.R.Rossi, "Colorimetry of total phenolics with phosphomolybdic phosphothungstic acid" Am. J. Enol. Vitic, 1965, Vol. (16), page : 144.

[5] L.Lagnika, "Etude phytochimique et activité biologique de substances naturelles isolées de plantes béninoises" Thèse de doctorat, Université Louis Pasteur, Strasbourg, 2005, page : 249. 
[6] J.Zhishen, T.Mengcheng, W.Jianming, "The determination of flavonoid contents in mulberry and their scavenging effects on superoxide radicals" J Food Chem, 1999, Vol. (64), page : 555 .

[7] D.Kim, O.Chun, Y.Kim, H.Moon, C.Lee, "Quantification of phenolics and their antioxidant capacity in fresh plums" J. Agric. Food Chem., 2003, Vol. (51), page : 6509.

[8] H.P.S.Makkar, "In Quantification of tannins in tree foliage" Working document, FAO/IAEA, Vienna, 2000.

[9] P.Schofield, D.M. Mbugua, A.N.Pell, "Analysis of condensed tannins" a review. Anim. Feed Sci. Technol. 2001, Vol. (91), page : 21.

[10] B.Sun, , JM.Richardo-da-Silvia, , I.Spranger, "Critical factors of vanillin assay for catechins and proanthocyanidins" J. of Agriculture and Food Chemistry, 1998, Vol. (46), page : 4267.

[11] R.Julkunen-Titto, "Phenolic constituents in the leaves of northem wiliows methods for the analysis of certain phenolics" Journal of Agricultural and Food chemistry, 1985, Vol.

(33), page : 213 .

[12] M.M.Giusti, R.E.Wrolstad "Anthocyanins: characterization and measurement with UVvisible spectroscopy" Current protocols in food analytical chemistry, New York: John Wiley \& Sons: Unit, 2001.

[13] J.G.Marco, "A rapid method for evaluation of antioxidants" Journal of the American Oil Chemists' Society, 1968, Vol. (45), page : 594.

[14] F.Deba, T.Dang Xuan, M.Yasuda, S.Tawata, "Chemical composition and antioxidant, antibacterial and antifungal activities of the essential oils from Bidens pilosa Linn. var. Radiata" Food Control, 2008, Vol. (19), page : 346.

[15] B.Tepe, M.Sokmen, HA.Akpulat, A.Sokmen, "Screening of the antioxidant potentials of six Salvia species from Turkey" Food Chem., 2006, Vol. (95), page : 200. 